\title{
Valeurs et projets par-delà la migration : recherche comparative sur des populations turques immigrantes et non immigrantes
}

\section{VALUES AND OBJECTIVES BEYOND THE MIGRATORY EXPERIENCE: COMPARATIVE RESEARCH INTO TURKISH IMMIGRANT AND NON-IMMIGRANT POPULATIONS}

\author{
Altay A. Manço
}

Volume 29, numéro 1, printemps 2000

URI : https://id.erudit.org/iderudit/010275ar

DOI : https://doi.org/10.7202/010275ar

Aller au sommaire du numéro

Éditeur(s)

Association des démographes du Québec

ISSN

0380-1721 (imprimé)

1705-1495 (numérique)

Découvrir la revue

Citer cet article

Manço, A. A. (2000). Valeurs et projets par-delà la migration : recherche comparative sur des populations turques immigrantes et non immigrantes. Cahiers québécois de démographie, 29(1), 33-55.

https://doi.org/10.7202/010275ar
Résumé de l'article

L'auteur étudie l'évolution des valeurs et des pratiques soscioculturelles au sein d'une population immigrée masculine en référence a une population non immigrée correspondante, au moyen d'une double démarche d'enquête par questionnaire exécutée parallèlement en Belgique et en Turquie, et apportant de l'information sur les pratiques matrimoniales, les représentations religieuses et les opinions relatives aux rôles et positions des femmes. L'expérience migratoire ne contraint pas les sujets à un repli culturel qui tendrait à renforcer les cadres de pensée et les éléments culturels de la région d'origine. Au contraire, on assiste à l'émergence d'une synthèse identitaire originale à visée adaptative, qui articule des éléments culturels divers provenant tant du pays d'origine que du pays d'accueil. 
Cahiers québécois de démographie

Vol. 29, no 1, printemps 2000, p. 33-55.

\title{
Valeurs et projets par-delà la migration : recherche comparative sur des populations turques immigrantes et non immigrantes
}

\author{
Altay A. MANÇO *
}

Notre démarche interdisciplinaire tente de conjuguer une interrogation en matière d'intégration sociale avec des questions sur la psychosociologie des groupes minoritaires. Cette approche suppose notamment, parmi les personnes issues des couches récentes de l'immigration en Europe occidentale, une dialectique identitaire polarisée par la conservation d'une "culture d'origine " et les exigences d'une assimilation socioéconomique à la société d'accueil (Camilleri et al., 1990).

Une hypothèse similaire, en termes d'équilibration entre tendances opposées, a été examinée en 1995 auprès d'un échantillon de jeunes hommes turcs installés en Belgique (Manço, 1998). L'objectif était de modéliser leurs comportements et attitudes à partir de l'observation d'un grand nombre de caractéristiques. Cette enquête fait ressortir plusieurs types

* Université de Liège, Groupe de recherches économique et sociale sur la population. Adresse: Clos des Coteaux 16 B/5001 Belgrade, Belgique. Courriel : altay.manco@skynet.be.Cette recherche a été réalisée avec le soutien de 1) l'Académie royale des sciences de Belgique, Classe des Lettres, Bourse de la Fondation Émile Waxweiler (1997), 2) la Communautê française de Belgique, Commissariat génêral aux relations internationales, et le ministère de l'Éducation nationale de la République de Turquie, Bourse conjointe de recherche scientifique (1998-1999).

Différentes personnes et institutions ont contribué à la réalisation de cette recherche. L'auteur souhaite les remercier ici pour leur soutien : Département de guidance et d'orientation psychologique de la Faculté d'éducation de l'Université du IX septembre à Buca-Izmir (Mme la Prof. Ferda Aysan), Municipalité d'Emirdağ (M. le Maire Ismet Güler), Municipalité de Davutlar (M. le Maire Adem Tuna), Agence de presse Info-Türk (Bruxelles, M. Doğan Özgüden), Mme Gonca Manço. 
de stratégies identitaires déterminées par les contextes socioéconomiques et familiaux, eux-mêmes en interaction avec une série de caractéristiques individuelles. Les stratégies identitaires des jeunes issus de l'immigration peuvent évoluer, sous l'effet d'une maturation et de facteurs contextuels, vers un état d'" équilibre dynamique " dont les effets s'exercent tant sur la scolarisation et l'insertion professionnelle que sur l'épanouissement de la personnalité.

Cette approche empirique s'est révélée opérante pour discriminer les divergences d'attitudes et de projets dans l'échantillon examiné. Les analyses factorielles ont fait ressortir quatre types de stratégies identitaires parmi les jeunes Turcs issus de l'immigration en Belgique. Il a donc été possible de définir l'interaction entre ces stratégies et la scolarisation, l'insertion économique ou l'épanouissement personnel : l'assimilation aux normes majoritaires n'est pas la seule stratégie de mobilité ascendante possible pour une minorité issue de l'immigration, la construction d'une identité "dialectique " correspond également à un degré de fonctionnalité.

Ainsi, les stratégies d'investissement de la culture d'origine par les migrants en Europe occidentale ne seraient pas nécessairement synonymes de marginalisation dans la société d'accueil, et l'investissement de la "culture d'origine " ne serait pas tant le fruit d'un repli identitaire dû au choc migratoire vécu par les immigrants, comme on le pense souvent, que le signe de l'adoption de stratégies de positionnement qui seraient en phase, notamment, avec l'évolution de l'opinion dans le pays d'origine, en l'occurrence la Turquie.

Or on sait (Güvenç, 1995) que l'opinion publique turque est elle-même traversée par des courants apparemment contradictoires (modernisation et mondialisation, mais également réislamisation) qui finissent par se combiner dans de multiples synthèses identitaires.

Comment l'évolution des valeurs socioculturelles au sein d'une population immigrée se situe-t-elle par rapport à l'évolution des valeurs et des projets observée dans le pays d'origine? Telle est donc la question de base que nous posons.

La réponse à cette question découlant d'observations sur la population turque immigree en Belgique peut contribuer à la recherche en matière d'accommodation et d'organisation sociales et éclairer la problématique de l'efficacité des stratégies identitaires des immigrés, qui mobilisent à la fois les ressources de la société d'origine et celles de la société d'accueil. 


\section{MISE EN PERSPECTIVE SCIENTIFIQUE}

Des investigations parfois anciennes ont porté sur les conduites et les caractéristiques respectives de groupes de migrants et de non-migrants d'une même origine. The Polish Peasant in Europe and America, de Thomas et Znaniecki (1918), première des études de ce type ${ }^{1}$, observe les migrants polonais avant, pendant et après leur départ pour les États-Unis. Des méthodes de recherche neuves pour l'époque, comme l'analyse qualitative de récits oraux ou l'étude d'un matériel écrit (presse, autobiographies, courrier privê) sont mises en œuvre.

Cette approche a permis, sans doute pour la première fois à cette échelle, d'intégrer aux données externes et objectives l'interprétation subjective que donnaient les immigrants polonais de leur expérience d'accommodation sociale : il était alors possible de concevoir certains liens entre changement social et changement des identités (Bastenier et Dassetto, 1993).

Plus près de nous, divers courants de recherches tentent d'évaluer les effets socioéconomiques sur certaines régions d'origine d'une émigration aussi massive que concentrée sur quelques points d'arrivée en Europe occidentale.

\section{Recherches à propos de l'émigration turque}

En Turquie, le fait migratoire a commencé, historiquement, dès les années 1950, avec l'exode rural (Alpay et Sariaslan, 1984; Köksal, 1986; Manço et Manço, 1992).

À l'intérieur d'un premier courant de recherches sociologiques, Kadioğlu (1994) a tenté d'analyser l'effet de l'émigration turque (en partie constituée de travailleuses ayant immigré seules) sur la conception des rôles sexuels au sein des familles, en comparant la situation des immigrantes pionnières, des femmes restées seules au pays d'origine et des femmes qui avaient rejoint leur mari immigrant, eu égard à un groupe témoin de femmes turques non exposées à l'immigration. Les résultats montrent une émancipation plus grande des femmes exposées à l'immigration, tout particulièrement dans le cas des travailleuses pionnières.

Représentant un deuxième courant de recherches, Gitmez (1983) a étudié l'effet du retour définitif des expatriés sur les régions d'origine. Il a en outre comparé les caractéristiques

1 Boski (1994) tente d'actualiser le même thème. 
socioéconomiques et culturelles des migrants de retour avec celles des émigrés dans leur ensemble : que pensaient les exmigrants de leur réinsertion dans leur pays d'origine ${ }^{2}$ ?

Un troisième courant s'est intéressé aux stratégies d'ajustement personnel (par exemple entre les valeurs d'accomplissement de soi et le collectivisme) face à la transition acculturative. La recherche s'est ici centrée tant sur les immigrants internes installés dans la périphérie populaire d'Istanbul que sur les immigrants externes installés, par exemple, dans les régions industrielles du Limbourg belge (Phalet et Claeys, 1993; Phalet et Hagendoorn, 1996).

La comparaison des modes de vie et de pensée avant et après la migration constitue une quatrième modalité de recherche. Pour déterminer le poids de la migration dans le changement social, ces recherches, fort nombreuses, confrontent habituellement les comportements et attitudes des migrants et des non-migrants d'une région d'origine donnée. Ainsi,

- Naess (1988) a décrit la vie religieuse et associative d'une communauté de chiites turcs (Alévis) dans son village d'origine (sud-ouest de la Turquie) et immigrés en Norvège.

- Boyer (1983) a comparé la socialité des Turcs immigrés dans le Périgord (France) avec celle des régions d'où ils sont originaires.

- Timmerman (1992) a étudié les différences et les ressemblances dans les stratégies de formation scolaire des jeunes filles turques à Istanbul, à Emirdağ et en Flandre.

- Enfin, Gailly (1992) et son équipe ont été à la base d'une étude sur les différences de perspective temporelle et de projets de vie entre jeunes Turcs de conditions sociales diverses vivant en Flandre ou à Istanbul.

Ces recherches montrent, en général, que la structuration identitaire est plus contradictoire et complexe chez les jeunes migrants que chez les non-migrants.

Dans ce courant d'investigations, certains chercheurs confrontent également les attitudes et comportements des personnes immigrées avec ceux qu'elles avaient avant l'immigration. Ainsi, Akhan (1992) s'intéresse aux comportements de maternage et aux pratiques entourant l'accouchement chez les femmes turques.

2 Une récente recherche d'Establet (1997) interroge d'anciens immigrés turcs en France réinstallés en Turquie, sur leurs visions et souvenirs de la France. 
Un cinquième et dernier courant de recherches concerne l'évolution socioéconomique des familles immigrées à l'étranger comparativement à celle des branches non immigrantes des mêmes familles ou des familles des régions d'origine. Cette vague de recherches est actuellement mise en ouvre par des équipes internationales de chercheurs (comprenant des scientifiques turcs), coordonnées par l'Institut néerlandais d'investigations démographiques (NIDI) en collaboration avec EUROSTATLuxembourg (Schoorl, 1998; van der Erf et Heering, 1994). L'objectif principal de ces travaux est de définir les causes des migrations internationales et d'identifier les facteurs importants qui génèrent les déplacements de populations pour des raisons économiques. L'évaluation du bien-être engendré par la migration est également au menu des préoccupations scientifiques.

Si la mise en route de telles investigations est des plus ardues, leur apport à l'analyse des effets conjoints de la migration, du changement socioculturel et du développement économique est à la mesure des efforts consentis.

Notre recherche personnelle, menée en Turquie, tente modestement de contribuer au développement de ces nouveaux courants de recherches sur l'immigration et ses multiples effets. Elle veut mettre en lumière des phênomènes d'accommodation et d'évolution sociales, l'évolution des cadres de pensée des populations - en immigration ou non - et le devenir conjoint des populations migrantes et non migrantes.

L'étude comparative des cadres de pensée de populations immigrees turques et de populations correspondantes non immigrantes nécessite une enquête sur les attitudes et valeurs des Turcs, attitudes et valeurs liées notamment à la notion de famille, à l'éducation des enfants, aux rapports entre les sexes, au travail, etc.

À ce propos, nous disposons de données importantes en ce qui concerne la population turque immigrée en Europe (de Tapia, 1995; Manço, 1998, etc.). En revanche, l'étude des mentalités ${ }^{3}$ en Turquie est un domaine encore peu exploité; du reste, les résultats de recherche qui existent sont peu diffusés dans les pays occidentaux.

3 “Une mentalité est le système de référence implicite d'un groupe social, homogène du point de vue de cet état d'esprit commun; ce système de référence lui permet de voir les choses d'une certaine manière et donc d'avoir des réactions et conduites en accord avec cette perception du monde "(Mucchielli, $1985: 5$ ). 


\section{Étude des attitudes culturelles et des mentalités en Turquie}

Sur les valeurs des adolescents, par exemple, il existe peu de données issues d'études comparatives internationales (travaux parfois générés par l'OCDE). Selon Offer et al. (1988), qui ont analysé l'image de soi de jeunes vivant dans huit pays différents, dont la Turquie, il existe chez les adolescents de tous les pays (développés ou en développement) un décalage relatif par rapport au cadre de pensée et d'action hérité de leurs parents. Or, si les jeunes sont bien en quête de nouvelles valeurs et d'une valorisation de soi en tant qu'individus, les chercheurs observent généralement que cette recherche identitaire va également de pair avec une intégration et une articulation progressives des représentations héritées des aînés.

Dans le domaine des valeurs associées à la famille, au mariage, à la conception et à l'éducation des enfants, ainsi qu'aux rôles sexuels, les travaux psychosociologiques de Kağitçibaşi (1991) font autorité. Ils interrogent les liens entre changement socioculturel et économique et adaptation psychologique des individus aux contextes changeants, notamment en situation d'industrialisation ou de migration (interne et externe). D'un point de vue plus général, Kağitçibaşi (1991: 10) tente de déterminer :

- si les modèles culturels définis par la "modernité " et par la " tradition " sont exclusifs l'un de l'autre;

- si des synthèses dialectiques, empiriquement descriptibles, sont possibles entre ces modèles.

Nous mentionnerons les résultats de certains travaux de Kağitçibaşi et de ses collaborateurs (notamment à propos des valeurs éducatives), en regard de nos propres données.

\section{OBJECTIFS ET MÉTHODE}

\section{Objet}

Pour comparer l'évolution des valeurs et des projets socioculturels au sein des populations immigrantes et non immigrantes, il faut réaliser en Turquie des études identiques à celles qui sont effectuées en Europe occidentale auprès de sujets immigrés ou issus de l'immigration turque.

Ces travaux auront pour objectif d'évaluer les attitudes, les projets, les valeurs et les comportements culturels dans divers 
domaines, comme les stratégies matrimoniales, les opinions concernant les femmes, les pratiques éducatives familiales, la consommation culturelle et la religiosité.

Notre recherche (Manço, 1998) effectuée en Belgique auprès d'immigrants turcs et de personnes de nationalité turque nées dans ce pays offre une possibilité de comparaison.

L'enquête comparative à effectuer en Turquie, pays d'origine, doit concerner des personnes non migrantes suffisamment nombreuses, choisies au hasard et présentant des caractéristiques générales semblables à celles de l'échantillon d'hommes turcs étudié en Belgique, pays d'immigration : on peut ainsi isoler, dans les êchantillons, les classes d'âge, le sexe et l'origine régionale.

Les résultats de la comparaison entre les données récoltées en Turquie et celles observées en Belgique permettront de définir les changements, les adaptations et les continuités, pardelà la migration, entre deux populations comparables et issues d'une même matrice socioculturelle.

\section{Sources de l'étude}

L'enquête organisée en Turquie pour répondre à cet objectif a donc concerné des personnes non migrantes (à l'exclusion de personnes rentrées au pays d'origine après une installation plus ou moins longue à l'étranger) présentant des caractéristiques comparables à celles de l'échantillon étudié en Belgique (hommes de 19 à 50 ans, essentiellement des travailleurs peu qualifiés, choisis aléatoirement...).

L'aire d'enquête est située à l'extrême est de la région égéenne. Il s'agit plus particulièrement d'Emirdağ ${ }^{4}$, petite entité semi-urbaine de la province d'Afyonkarahisar, sise à environ 300 kilomètres au nord-est d'Izmir et à quelque 200 kilomètres au sud-ouest d'Ankara: près de 31 pour cent des Turcs immigrés en Belgique sont originaires cle cette seule localité (Manço, 1998).

Les entretiens ont été réalisés sur la base d'un formulaire de 84 points, pour la plupart extraits du questionnaire - plus long - "Histoire migratoire et mobilité sociale" (1994) produit à l'occasion d'une recherche interuniversitaire coordonnée par: le Département de sociologie de la Vrije Universiteit de Bruxelles (voir plus loin).

4 Voir la carte 1 et la section suivante. 


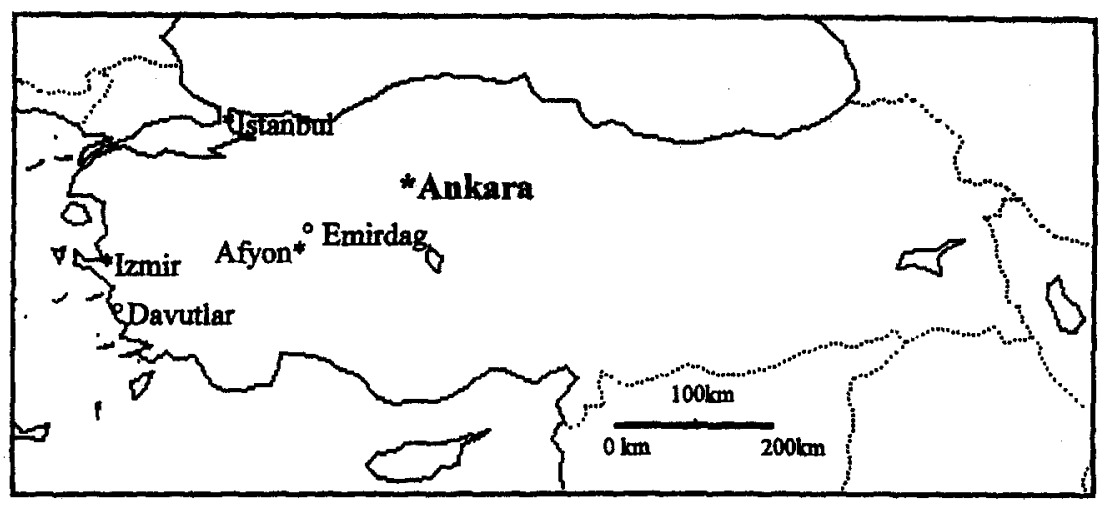

CARTE 1 - Situation géographique des lieux cités

Le questionnaire à choix multiple ainsi établi a servi à évaluer les attitudes et comportements culturels (valeurs religieuses, familiales...) et à identifier les projets d'avenir (personnels et éducatifs) des personnes rencontrées à Emirdağ. Il a également permis de définir les caractéristiques sociodémographiques des ménages, notamment l'appartenance socioéconomique et le niveau de formation des sujets et de leur père. Les liens avec l'étranger (famille immigrée en Europe...) font aussi partie des domaines investigués.

Les interviews ont été réalisées grâce à la collaboration des municipalités locales (contactées de longue date). Concrètement, des agents communaux formés à cet effet ont réalisé les interviews, le chercheur supervisant les opérations.

L'enquête réalisée à Emirdağ a permis d'interroger 93 hommes de 19 à 50 ans, tous originaires de cette localité, vivant de manière permanente en Turquie et n'ayant jamais voyagé à l'étranger. Ces personnes ont été choisies aléatoirement et abordées pour un entretien dans des lieux publics fréquentés par une population tout venant, comme l'administration municipale ou le marché public hebdomadaire. Les enquêteurs ont posé les questions et coché les réponses. Le travail de terrain s'est effectué en octobre 1998. L'échantillon est numériquement limitê. Les résultats présentés ici doivent par conséquent être considérés à titre indicatif.

L'enquête réalisēe en Belgique ${ }^{5}$ a été coordonnée par le professeur Ron Lesthaeghe du Centrum voor sociologie de

5 Pour les informations méthodologiques concernant l'enquête réalisée en Belgique, voir Manço (1998 ; 46-48). 
l'Université libre flamande de Bruxelles (1994-1996). Elle porte sur la population masculine turque installée en Belgique et fait suite à une autre étude de la même université consacrée à la population féminine turque installée en Flandre et à Bruxelles. Le questionnaire développé à l'occasion de l'enquête comporte 419 éléments couvrant divers domaines : histoire migratoire, famille d'origine, données démographiques, maitrise linguistique, formation (post-)scolaire, carrière et projets professionnels, attitudes et valeurs culturelles, etc. L'outil d'enquête est disponible en deux versions (turc-français et turc-néerlandais).

L'échantillon compte 1462 individus de nationalité turque de plus de 19 ans, choisis aléatoirement sur la base de listes administratives. Plus de 150 enqueteurs mascullins turcs ont été formés pour procéder à l'application du questionnaire; un nombre appréciable d'interviews ont été réalisés dans la langue d'origine des répondants.

$\mathrm{Au}$ total, pour la tranche d'âge donnée, un homme turc sur 20 a été contacté dans les régions citées, entre le mois de novembre 1994 et le mois de mai 1995. Les sujets ont été rencontrés le plus souvent à leur domicile et sur rendez-vous ${ }^{6}$.

Dans la présente recherche ont été envisagées exclusivement les données concernant :

- la population de 19 à 50 ans originaire d'Emirdağ et ayant immigré en Belgique après l'âge de 14 ans $(N=289)^{7}$ et

- le groupe des personnes nées en Belgique de parents immigrês turcs, et ayant toujours vécu en Belgique $(\mathrm{N}=172)$.

L'êchantillon des sujets turcs de Belgique est composé de personnes rencontrées dans différentes communes, à Bruxel-

6 Au total, 2019 visites ont été réalisées par les enquêteurs auprès de ressortissants adultes turcs de plus de 19 ans (891 en Flandre, 635 à Bruxelles et 493 en Wallonie). Finalement, 1462 interviews utilisables ont èté recueillies (729 en Flandre, 413 à Bruxelles et 321 en Wallonie), dont 989 avec des personnes de 19 à 50 ans. Cela conduit à constater un taux de réalisation global de 72 pour cent (82 pour cent en Flandre, 65 à Bruxelles et 65 en Wallonie). Les motifs de retour ou de non-réponse les plus courants sont les absences répétées ou prolongées des sujets (132 cas), les déménagements ou les erreurs d'adresse (137 cas), les refus formels de participer à l'étude ( 220 cas), les maladies, les hospitalisations, l'état de déficience mentale de quelques personnes tirées au hasard, les décès (6 cas) et l'incarcération de certains sujets (10 cas). L'équipe de contrôle a également dũ annuler 14 interviews douteux ou frauduleux.

7 L'enquête permet de savoir que le dernier lieu habité en Turquie, avant l'émigration vers la Belgique, est bien Emirdağ, pour tous les sujets de cette catégorie. 
les, en Wallonie et en Flandre. Les personnes immigrées en Belgique avant l'âge de 14 ans et les personnes âgées de plus de 50 ans au moment de l'enquête ont donc été exclues des calculs statistiques pour des raisons de comparabilité avec l'échantillon interrogé à Emirdağ. Il en est de même, bien entendu, de l'immigration venant d'autres régions de la Turquie.

Les résultats sont donnés séparément pour chaque catégorie envisagée : migrants, non-migrants et personnes nées en immigration. Cette dernière catégorie correspond au cas des personnes nées en Belgique et éduquées par des parents turcs immigrés, mais n'ayant jamais vécu en Turquie : il s'agit d'une classe à part entière, dont la confrontation aux autres est riche d'enseignements sur les processus d'acculturation (tableau 1).

TABLEAU 1 - Échantillons

\begin{tabular}{|c|c|c|}
\hline $\begin{array}{l}\text { Personnes d'Emirdağ } \\
\text { non migrantes }\end{array}$ & $\begin{array}{l}\text { Personnes d'Emirdağ } \\
\text { immigrées en Belgique }\end{array}$ & $\begin{array}{l}\text { Personnes nées } \\
\text { en Belgique de } \\
\text { parents turcs }\end{array}$ \\
\hline $\mathrm{N}=93$ & $N=289$ & $\mathrm{~N}=172$ \\
\hline $\begin{array}{l}\text { Personnes de } 19 \text { à } \\
50 \text { ans nées à } \\
\text { Emirdağ et y ayant } \\
\text { toujours vécu, et } \\
\text { n'ayant jamais voyagé } \\
\text { à l'étranger }\end{array}$ & $\begin{array}{l}\text { Personnes de } 19 \text { à } 50 \text { ans } \\
\text { nées à Emirdağ et y } \\
\text { ayant vécu jusqu'à } \\
14 \text { ans, et immigrées en } \\
\text { Belgique après l'âge de } \\
14 \text { ans }\end{array}$ & $\begin{array}{l}\text { Personnes jeunes } \\
\text { de plus de } \\
19 \text { ans, nées en } \\
\text { Belgique et y } \\
\text { ayant toujours } \\
\text { vécu }\end{array}$ \\
\hline
\end{tabular}

\section{EMIRDAĞ, LA ZONE D'ORIGINE}

Emirdağ est une municipalité semi-rurale de taille moyenne de quelque 22000 habitants. Pendant les mois d'été, sa population atteint environ 60000 unités, car les émigrés originaires de la région sont très nombreux à y revenir pour les vacances annuelles. Selon les responsables municipaux, cela ne va pas sans poser des problèmes d'organisation ${ }^{8}$ et d'approvisionnement, notamment en eau. Emirdağ est, de fait, située dans une région semi-aride. Économiquement peu développée, elle recèle

8 Les autorités municipales nous ont expressément demandé d'exécuter notre étude en dehors de la période estivale; cela convenait parfaitement à notre objectif de n'interroger que des personnes effectivement installées en Turquie. 
somme toute très peu de richesses naturelles ${ }^{9}$. La municipalité, les autres administrations et. le secteur de l'enseignement (présence d'écoles supérieures techniques), par exemple, sont les plus gros pourvoyeurs d'emploi du district. Cette situation économique explique largement la poussée migratoire interne et internationale observée dans cette région (den Exter et Kutlu, 1992).

Selon Hall (1995), l'est de la région égéenne a fourni près de 16 pour cent de l'émigration ouvrière turque entre 1961 et $1975^{10}$, soit environ 300000 travailleurs, auxquels il faut ajouter les membres de leur famille ayant immigré. Plus du quart de cet effectif serait en fait parti avec un statut de "touriste ", dans l'espoir de régulariser sa situation une fois sur place, avec l'aide de proches déjà installés en Europe. Cette proportion de "semi-clandestins" est l'une des plus fortes observees en Turquie. Elle explique en partie pourquoi certaines communautés locales se reconstituent dans certaines régions européennes d'immigration ${ }^{11}$.

Gitmez (1983) évalue également à environ 22 pour cent la proportion d'émigrants "clandestins " (sans contrat d'emploi préalable à l'émigration) originaires d'Afyon (voir la carte 1). En outre, il compte, parmi ces émigrants, trois personnes originaires des villages périphériques (comme Emirdağ, par exemple) pour une personne issue des entités urbaines, comme la ville d'Afyon, chef-lieu de province.

9 Selon les données de Gitmez (1983), la province d'Afyon (ou Afyonkarahisar) se situe au 36e rang sur 67 sur l'échelle interprovinciale de développement socio-économique de la Turquie. Cet index est calculé par le Bureau du Plan turc, à partir de 34 indices de développement, comme le taux d'alphabétisation, le nombre de médecins par habitant, le nombre d'automobiles au kilomètre carré, le produit intérieur brut, etc. Or, le district d'Emirdağ se révèle particulièrement peu favorisé comparé à d'autres localitês de la province d'Afyon bénéficiant, notamment, dune plus grande densité de commerces et d'une industrie du marbre.

10 Arrêt officiel de limportation de travailleurs dans la plupart des pays européens.

11 En ce qui concerne Emirdağ et la présence massive de personnes de cette région en Belgique, les hommes ayant connu les premières heures de l'émigration vers l'Europe parlent de l'effet facilitateur qu'aurait eu, dans les années 1960-1970, le personnel de l'Ambassade de Belgique à Ankara (ville relativement proche) soucieux de répondre aux appels pressants d'industriels belges à court de main-d'œuvre. Ainsi, on évoque des séances d'information et de recrutement organisées à l'époque, à Emirdağ, par les membres de l'Ambassade de Belgique. 
Les émigrants originaires d'Emirdağ se répartissent dans l'ensemble des pays où sont présents les travailleurs immigrés turcs et leur famille, à commencer, bien sûr, par l'Allemagne (où vivent les trois quart des Turcs d'Europe) : on note également la présence des gens d'Emirdağ aux Pays-Bas, en France et au Danemark (de Tapia, 1995; Manço et Manço, 1992). La Belgique ne reçoit finalement qu'une petite partie des émigrants d'Emirdağ, mais ces derniers y sont particulièrement concentrés, notamment dans les communes de Schaerbeek et de Saint-Josse-ten-Noode (Reniers, $1997: 10$ ).

On trouvera une bonne synthèse sur les contextes socioéconomique, démographique et ethnologique d'Emirdağ dans den Exter et Kutlu (1992), qui tentent également de mesurer les effets de l'émigration massive sur le potentiel de développement de cette région de l'Anatolie ${ }^{12}$. Notons que cette localité n'apparaît en aucun cas comme représentative des valeurs socioculturelles de la Turquie ${ }^{13}$.

\section{RÉSULTATS DE LA RECHERCHE}

\section{Caractéristiques sociodémographiques des échantillons}

Les deux échantillons qui nous concernent directement ne comprennent que des hommes turcs choisis au hasard et originaires de la région d'Emirdağ; certains ont immigré en Belgique après l'âge de 14 ans, d'autres sont restés au pays. Le groupe interrogé en Belgique a la mème structure par âge que celui d'Emirdağ (tableau 2). Parmi les personnes interrogées en Belgique figurent 172 sujets nés dans ce pays, et qui ne sont donc pas des migrants. Certains des parents de ces jeunes (environ un sur trois) qui sont de la "deuxième génération" sont eux aussi originaires de la région d'Emirdağ. Les observa-

12 L'article de den Exter et Kutlu (1992) propose aussi une analyse qualitative des comportements (notamment économiques) des émigrants et des non-émigrants.

13 En témoigne notamment un travail effectué par nous sur la municipalité de Davutlar, petite commune côtière semi-rurale où les pratiques matrimoniales sont moins traditionnelles qu'à Emirdağ (comme nous le verrons) et le taux de pratique religieuse moins élevé. Les habitants de Davutlar semblent davantage porteurs de valeurs éducatives attachées à la notion d'êmancipation individuelle que ceux d'Emirdağ, plus orientés vers des valeurs collectives et familiales. Les conceptions sur l'éducation des jeunes filles, par exemple, distinguent fortement les deux localités. Pour de plus amples comparaisons entre Davutlar et Emirdağ, voir Manço, 1999. 
TABLEAU 2 - Structure par âge des échantillons

\begin{tabular}{lrrrrrr} 
& \multicolumn{2}{c}{ Emirdağ } & \multicolumn{2}{c}{ Immigrês en Belgique } & \multicolumn{2}{c}{ Nés en Belgique } \\
Âges & \multicolumn{1}{c}{ N } & \multicolumn{1}{c}{$\%$} & $\mathrm{~N}$ & $\%$ & $\mathrm{~N}$ & \multicolumn{1}{c}{$\%$} \\
\hline $19-28$ & 49 & 53 & 152 & 52 & 167 & 97 \\
$29-38$ & 30 & 33 & 93 & 32 & 5 & 3 \\
$39-48$ & 9 & 9 & 28 & 10 & 0 & 0 \\
$49-50$ & 5 & 5 & 16 & 6 & 0 & 0 \\
Totaux & 93 & 100 & 289 & 100 & 172 & 100 \\
\hline
\end{tabular}

tions réalisées auprès de ce groupe, présentées à part, permettent une illustration des phénomènes de changement culturel dans des contextes migratoires variés. Naturellement, compte tenu de l'histoire migratoire turque vers l'Europe, ces personnes sont plus jeunes que celles des autres catégories de l'étude.

L'échantillon tiré en Belgique est essentiellement composé de personnes de qualification relativement faible, c'est-à-dire ayant un diplôme d'études primaires ou secondaires inférieures, souvent obtenu dans une section professionnelle (Manço, 1998). Le niveau de scolarité des personnes non émigrées (tableau 3, échantillon d'Emirdağ) est nettement plus élevé que celui des personnes qui ont migré. Cette situation s'explique par la sélectivité des phénomènes migratoires, qui ont surtout mobilisé des travailleurs peu qualifiês. De plus, les immigrés n'ont pas trouvé au pays d'accueil un contexte sociologique favorable à leur formation, ne serait-ce qu'à cause de leurs difficultés d'ordre linguistique. Le groupe des jeunes nés en immigration présente un profil intermédiaire entre les deux autres catégories. Cela ressort surtout quand on comptabilise la proportion de personnes ayant dépassé le niveau d'études qui correspond à la fin de l'obligation scolaire, dans les deux pays qui nous concernent ${ }^{14}$. À peine 11 pour cent des Turcs installés en Belgique ont poursuivi leur scolarité au-delà de

14 Depuis 1985, l'obligation scolaire concerne, en Belgique, les enfants de 6 à 18 ans. La fin de cette obligation correspond, normalement, à la fin du cycle secondaire supérieur. Avant cette date, la scolarité était obligatoire jusqu'à 16 ans (fin normale des études secondaires inférieures). En Turquie, l'obligation scolaire concernait, jusqu'il y a peu, les enfants de 7 à 12 ans et correspondait à la fin des études primaires. Depuis quelques années, la limite supérieure de cette obligation a été portée à 15 ans (études secondaires inférieures). Cependant, compte tenu de l'âge moyen des échantillons rencontrés en Turquie et en Belgique, nous n'avons pas tenu compte de ces évolutions récentes. 
TABLEAU 3 - Niveaux de formation dans les échantillons (\%)

\begin{tabular}{lrrr}
\hline Catégorie & $\begin{array}{c}\text { Emirdağ } \\
(\mathrm{N}=93)\end{array}$ & $\begin{array}{r}\text { Immigrés en Belgique } \\
(\mathrm{N}=289)\end{array}$ & $\begin{array}{c}\text { Nés en Belgique } \\
(\mathrm{N}=172)\end{array}$ \\
\hline Aucun diplôme & 3 & 2 & 0 \\
Primaire & 34 & 48 & 24 \\
Second. inférieur & 6 & 39 & 65 \\
Second. supérieur & 42 & 10 & 10 \\
Supérieur & 15 & 1 & 1 \\
Totaux & 100 & 100 & 100 \\
\hline
\end{tabular}

Note $: \chi^{2}=114,81, \mathrm{p}<0,00001$ (les valeurs surreprésentees sont en italiques).

l'obligation scolaire et obtenu un certificat d'études (personnes immigrées et nées en Belgique). Cette proportion est de 63 pour cent dans l'échantillon interrogé en Turquie.

On constate également une différence de niveau de vie entre les Turcs de Belgique et ceux d'Emirdağ : 60 pour cent des immigrés affirment être propriétaires d'une habitation, comparativement à 33 pour cent pour l'échantillon d'Emirdağ. Ce résultat confirme l'impact pour les immigrés d'avoir pu travailler dans un pays développé. Seulement 18 pour cent des sujets nés en Belgique sont dans cette situation, mais ils sont aussi beaucoup plus jeunes $\left(\chi^{2}=38,87, p<0,00001\right)$.

\section{Pratiques matrimoniales}

Parmi les 93 sujets interrogés à Emirdağ, 62 (66 pour cent) sont mariés, comparativement à 273 ( 94 pour cent) dans l'échantillon des immigrés de Belgique. Seulement 41 pour cent des 172 personnes nées en Belgique (qui constituent un groupe plus jeune) sont mariées $\left(\chi^{2}=63,59, \mathrm{p}<0,00001\right)$.

Ce constat tend à montrer que l'âge au mariage est plus tardif pour les hommes en Turquie; ce fait peut s'expliquer par les différences de niveau de vie entre les deux régions comparées. Les immigrants pourraient également accorder plus d'importance au mariage parce que celui-ci constitue une voie d'arrivée légale en Belgique pour des jeunes de Turquie, en particulier des jeunes proches des familles déjà immigrées (Manço, 1998). Les faits suivants appuient cette hypothèse :

- Plus de 44 pour cent des mariages concernant les Turcs immigrés de Belgique sont conclus entre personnes issues de la même famille. Ce taux est de 29 pour cent seulement 
à Emirdağ. Cinquante-deux pour cent des jeunes nés en Belgique sont mariés à des personnes de leur famille $\left(\chi^{2}=11,22, p=0,0037\right)$. Par ailleurs, à Emirdağ et en Belgique, 16 et 24 pour cent respectivement des mariages concernent des cousins (fils ou fille de la tante ou fils ou fille de l'oncle, en particulier paternels). Pour les jeunes mariés nés en Belgique cette valeur est de 36 pour cent $\left(\chi^{2}=10,71, p=0,0047\right)$.

- En immigration, 90 pour cent des répondants immigrés ou nés en Belgique pensent qu'il serait important de choisir un gendre dans leur famille s'ils devaient marier leur fille; une telle réponse n'est donnée que par 47 pour cent seulement des personnes rencontrées à Emirdăg $\left(\chi^{2}=66,95\right.$, $\mathrm{p}<0,00001$ ).

Ces fortes différences soulignent la résurgence des pratiques traditionnelles en situation migratoire, en raison de l'occasion favorable que représente le mariage pour des personnes qui souhaitent s'installer en Europe.

En Belgique, 75 pour cent des répondants immigrés déclarent, en revanche, que leur mariage s'est surtout conclu, en définitive, grâce aux initiatives propres des futurs époux. Pareille réponse n'est donnée que par 61 pour cent des personnes rencontrées à Emirdağ, laissant entendre une plus grande implication des parents (en particulier des parents de l'époux) dans l'arrangement du mariage. Chez les jeunes mariés nés en Belgique, la proportion des personnes qui déclarent que leur mariage s'est conclu grâce à leurs propres initiatives est de 82 pour cent $\left(\chi^{2}=11,51, p=0,0032\right)$.

\section{Rapports aux femmes}

Les hommes turcs interrogés en Belgique et à Emirdağ pouvaient se prononcer sur cinq propositions concernant les rôles et positions des femmes dans la vie sociale et familiale (tableau 4).

Les rêpondants questionnés en Turquie ont des opinions plus traditionnelles : 54 pour cent sont tout à fait contre l'idée qu'une femme parle à des hommes inconnus de son époux. Seulement 24 pour cent des hommes turcs immigrés en Belgique et 26 pour cent des jeunes Turcs nés en Belgique sont de cet avis.

De la même manière, 50 pour cent des hommes d'Emirdağ sont " tout à fait d'accord " ou " plutôt d'accord " avec la deuxiè- 
TABLEAU 4-Représentations concernant les femmes dans les échantillons (totaux verticaux $=100 \%$ )

\begin{tabular}{|c|c|c|c|c|c|c|c|c|c|c|c|c|c|c|c|}
\hline \multirow{2}{*}{$\begin{array}{l}\text { Énoncés } \rightarrow \\
\text { Catég. } \rightarrow\end{array}$} & \multicolumn{3}{|c|}{1} & \multicolumn{3}{|c|}{2} & \multicolumn{3}{|c|}{3} & \multicolumn{3}{|c|}{4} & \multicolumn{3}{|c|}{5} \\
\hline & $\mathrm{E}$ & IB & NB & $\mathrm{E}$ & IB & NB & $\mathrm{E}$ & IB & $\mathrm{NB}$ & $\mathrm{E}$ & IB & $\mathrm{NB}$ & $\mathrm{E}$ & IB & NB \\
\hline $\begin{array}{l}\text { Pas du tout } \\
\text { d'accord }\end{array}$ & 54 & 24 & 26 & 32 & 20 & 25 & 27 & 16 & 28 & 17 & 2 & 2 & 19 & 3 & 3 \\
\hline $\begin{array}{l}\text { Plutôt pas } \\
\text { d'accord }\end{array}$ & 13 & 29 & 26 & 9 & 29 & 35 & 10 & 20 & 30 & 10 & 7 & 9 & 7 & 7 & 3 \\
\hline $\begin{array}{l}\text { Difficile de } \\
\text { choisir }\end{array}$ & 11 & 19 & 25 & 9 & 18 & 20 & 14 & 17 & 19 & 13 & 18 & 23 & 14 & 14 & 11 \\
\hline $\begin{array}{l}\text { Plutôt } \\
\text { d'accord }\end{array}$ & 10 & 21 & 15 & 18 & 23 & 12 & 19 & 30 & 13 & 22 & 47 & 44 & 17 & 49 & 52 \\
\hline $\begin{array}{l}\text { Tout à fait } \\
\text { d'accord }\end{array}$ & 12 & 7 & 8 & 32 & 10 & 8 & 30 & 17 & 10 & 38 & 26 & 22 & 44 & 27 & 31 \\
\hline
\end{tabular}

Énoncés

1. "Une femme peut tranquillement parler avec des hommes, mẽme si son mari ne les connait pas ". $\chi^{2}=33,51, p<0,00001$.

2. "Lorsque des hommes étrangers viennent en visite, les femmes se retirent de préférence dans une autre pièce ". $\chi^{2}=77,16, p<0,00001$.

3. "Lorsqu une femme quitte sa maison, elle doit porter un foulard sur la tête . $\chi^{2}=32,54, p<0,00001$.

4. "En plus de son travail ménager, une femme doit se mêler à la vie en société et jouer un rôle important dans le domaine de la religion et de la politique ". $\chi^{2}=39,48, p<0,00001$.

5. "La femme doit avoir le droit d'aller travailler en dehors de la maison ". $\chi^{2}=46,35, \mathrm{p}<0,00001$.

Les valeurs surreprésentées sont en italiques.

\section{Catégories}

$E$ : nès et demeurés à Emirdağ $(N=93)$; IB : immigrès en Belgique $(N=289)$;

$\mathrm{NB}$ : nés en Belgique $(\mathrm{N}=172)$.

me proposition ("Lorsque des hommes étrangers viennent à la maison, les femmes se retirent dans une autre pièce ». Cette valeur, calculée de la même manière, est en Belgique de 33 pour cent pour les immigrés et de 20 pour cent seulement pour les personnes nées en immigration.

On constate également que les hommes interviewès en Turquie sont plutôt favorables au port du foulard à l'extérieur. C'est le point de vue inverse qui prévaut parmi les Turcs nés en Belgique.

Des différences importantes sont également relevées à propos des représentations liées aux rôles socioéconomiques 
des femmes dans la société. Ainsi, parmi les Turcs immigrés en Belgique, 73 pour cent sont d'accord avec l'idée que les femmes doivent jouer un rôle important dans les domaines de la religion et de la politique (énoncé 4). Parmi les jeunes nés en Belgique, ce point de vue recueille 66 pour cent des suffrages. À Emirdağ, 60 pour cent des hommes de notre échantillon adhèrent à cette proposition; 27 pour cent s'y opposent.

En Belgique, la grande majorité ( 83 pour cent) des jeunes hommes turcs nés en immigration sont d'accord ou tout à fait d'accord avec la proposition "La femme doit avoir le droit d'aller travailler en dehors de la maison ". Auprès des hommes interrogès à Emirdağ et auprès des immigrés en Belgique, cette idée ne recueille, en revanche, que 61 et 76 pour cent respectivement des suffrages. À Emirdağ, un homme sur 5 s'oppose fermement au travail professionnel de la femme.

À propos de cette dernière proposition, on peut signaler à titre indicatif que, lors d'une enquēte exécutée en 1988 par l'institut de sondage turc PI-AR, 82 pour cent des 2007 jeunes interrogés - représentatifs de la population turque de 15 à 26 ans - pensaient que " la femme a le droit d'aller travailler en dehors de son ménage ". En revanche, une étude du Bureau du Plan turc (Devlet Planlama Teşkilati) réalisée en 1991 auprès de 18210 ménages représentatifs de la population générale montre que 62 pour cent des hommes adultes et 42 pour cent des femmes adultes sont contre l'idée du travail professionnel de la femme ${ }^{15}$. Cette dernière recherche souligne l'importance des écarts, en ce qui concerne les conceptions sur l'emploi féminin, entre les espaces urbains et les espaces ruraux, d'ordinaire plus traditionalistes (sources citées par l'hebdomadaire Cumhuriyet-Hafta du 8 novembre 1991).

Enfin, selon Erder (1984), 39 pour cent des répondants d'un échantillon représentatif de la population adulte turque $(N=541)$ sont contre le droit au travail de la femme. Cette proportion est de 49 pour cent parmi les ouvriers, 39 pour cent parmi les paysans et 18 pour cent seulement parmi les étudiants universitaires.

Dans notre étude, 19 pour cent des hommes mariés d'Emirdağ, 40 pour cent des hommes immigrés en Belgique et 25 pour cent des jeunes nés dans ce pays ont une épouse qui travaille à l'extérieur du ménage $\left(\chi^{2}=11,61, p=0,003\right)$.

15 Selon les données de l'Institut de statistique de l'État turc, 33 pour cent des femmes de plus de 12 ans sont actives (1991). 
On constate donc une opinion et des comportements plus favorables au travail professionnel des femmes dans le groupe masculin turc installé en Belgique que dans le groupe correspondant vivant en Turquie. Dans ce pays, par ailleurs, les représentations à propos du travail des femmes semblent évoluer, depuis une décennie au moins, dans le sens d'une plus grande acceptation. À vrai dire, il existe d'importantes distorsions entre les positions des hommes et des femmes, des jeunes et des adultes, des citadins et des ruraux, et des différents groupes socioprofessionnels.

\section{Éducation des enfants}

Quel que soit le pays d'enquête, plus de la moitié des répondants estiment qu'il est important qu'un garçon "sache penser seul et s'intéresse au comment et au pourquoi des choses ". En revanche, plus de 60 pour cent des sujets, dans les deux pays, pensent qu'il est important qu'une fille "ait de bonnes manières et obéisse à ses parents ".

La comparaison des données récoltées en Turquie et en Belgique ne permet donc pas, à ce niveau, d'observer des diffërences significatives.

Une recherche déjà ancienne de Kağitçibaşi (1991), réalisée à la fin des années 1970 , montre également que 60 pour cent des 1040 personnes d'un échantillon représentatif de la population adulte turque estiment que l'obéissance est la valeur éducative la plus importante. Dans cet échantillon, à peine 18 pour cent des sujets privilégient l'indépendance comme objectif de l'éducation familiale.

Il est donc possible de parler d'une certaine stabilité des valeurs pour ces aspects de l'éducation familiale.

\section{Religiosité}

L'analyse des questions concernant les représentations liées à la religion permet de souligner l'importance accordée à la religion par la communauté turque de Belgique (tableau 5). En effet, parmi les Turcs immigrés, 90 pour cent sont tout à fait d'accord ou d'accord avec la proposition "La religion est une chose très importante dans ma vie ". Dans le groupe de personnes rencontrées à Emirdağ, par contre, les sujets qui donnent la mème réponse représentent 70 pour cent de 
TABLEAU 5-Représentations et pratiques religieuses dans les échantillons

\begin{tabular}{|c|c|c|c|c|c|c|c|c|c|c|c|c|c|}
\hline Énoncés $\rightarrow$ & & 6 & & & 7 & & & 8 & & Énoncé & & 9 & \\
\hline atég. $\rightarrow$ & $\mathrm{E}$ & IB & NB & $\mathrm{E}$ & IB & NB & E I & IB $\mathrm{N}$ & NB & atégorie & E & IB & NB \\
\hline & ${ }_{16}$ & 7 & 13 & 10 & 6 & 7 & 12 & 1 & 8 & & 6 & 9 & \\
\hline & 3 & 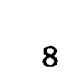 & 3 & 6 & 5 & 4 & 9 & 2 & 8 & & 33 & 13 & 36 \\
\hline & 14 & 14 & 11 & 16 & 12 & 11 & 9 & 7 & 7 & & 10 & 36 & 9 \\
\hline utôt & 25 & 40 & 28 & 24 & 43 & 24 & 15 & 30 & 17 & & 33 & 26 & 27 \\
\hline & 42 & 31 & 45 & 47 & 34 & 54 & 55 & 60 & 60 & $\begin{array}{l}\text { lus } \\
\text { souvent }\end{array}$ & 18 & 16 & 2.1 \\
\hline $\operatorname{taux}$ & 100 & 100 & 1001 & 1001 & 1001 & 1001 & 1001 & 10010 & 100 & Totaux & 100 & 100 & 100 \\
\hline
\end{tabular}

Énoncés

6. " La religion et la prière me procurent d'abord le réconfort dans les moments où le malheur me touche ou lorsque je suis triste. "N.S.

7. "Par ma religion et ma prière, je suis protégé du mal. "N. S.

8. "La religion est une chose très importante dans ma vie." $\chi^{2}=20,44, p=0,0088$.

9. "Fréquentez-vous la mosquée ? " $\chi^{2}=39,33, \mathrm{p}<0,00001$.

Les valeurs surreprésentées sont en italiques.

Catégories

$E$ : nés et demeurés à Emirdağ $(N=93) ;$ IB : immigrés en Belgique $(N=289)$; NB : nés en Belgique $(N=172)$.

l'effectif. On n'observe pas de différences significatives pour les autres points destinés à sonder le sens accordé à la religiosité.

Le taux de pratique religieuse est paradoxalement moins important parmi les sujets issus de l'immigration que parmi les personnes installées en Turquie. Ainsi, 51 pour cent des hommes d'Emirdağ, mais 42 pour cent des Turcs immigrés en Belgique, fréquentent la mosquée au moins une fois par semaine (tableau 5). Par ailleurs, 93 pour cent des musulmans rencontrés en Turquie affirment respecter le jeûne du mois de Ramadan, comparativement à 85 pour cent de l'échantillon immigré et à 75 pour cent de l'échantillon des jeunes nés en immigration $\left(\chi^{2}=12,31, \mathrm{p}=0,0021\right)$. 


\section{CONCLUSION}

Nous avons tenté de dêterminer le sens de l'évolution des valeurs et pratiques socioculturelles au sein d'une population immigrée en référence à une population non immigrée correspondante, au moyen d'une double démarche d'enquête exécutée en Belgique et en Turquie. Malgré une série de difficultés méthodologiques, un faisceau de constats peut être retenu.

Les pratiques matrimoniales revêtent une importance particulière en immigration et se traduisent par des comportements davantage traditionnels que dans le pays d'origine: mariage arrangé avec des familiers, âge au mariage plus jeune qu'en Turquie... En revanche, les personnes interrogées en Belgique affirment, paradoxalement, avoir joué dans la conclusion de leur mariage un rôle individuel plus important que les sujets rencontrés en Turquie, dont le mariage semble être davantage orienté par la volonté de leurs parents. Ces constatations sont particulièrement vraies pour les jeunes nés de parents immigrés.

Un constat parallèle concerne les pratiques et les représentations religieuses. Les Turcs de Belgique ont un niveau de pratique et d'observance religieuse plus faible que les hommes interviewés à Emirdağ, dans le cas du jeûne rituel par exemple. Par contre, les immigrés accordent une importance plus grande à la religion et à la vie religieuse en tant qu'éléments de référence morale dans leurs expériences d'exil.

Enfin, eu égard aux rôles et positions des femmes, les hommes turcs rencontrés en Belgique se montrent moins traditionnels que leurs homologues non immigrés: ils attribuent aux femmes des rôles importants dans la vie socioprofessionnelle, politique et religieuse, contrairement aux hommes interrogés en Turquie, selon lesquels il convient d'attribuer aux femmes un rôle limité surtout au domaine familial et un statut subordonné au mari.

L'expérience migratoire ne force donc pas les sujets à adopter une position de repli culturel tendant à renforcer les cadres de pensée et les éléments culturels de la région d'origine. La situation décrite ici témoigne plutôt de l'émergence d'une synthèse identitaire originale à visée adaptative, qui articule des éléments culturels divers provenant tant du pays d'origine que du pays d'accueil.

Ainsi, alors que les pratiques religieuses se sécularisent et que les représentations concernant les rapports entre les sexes 
se libéralisent, on assiste, en même temps, à un renforcement des pratiques matrimoniales traditionnelles et du sens du sacré, qui représentent, dans le contexte migratoire, des éléments culturels fonctionnels.

\section{RÉFÉRENCES BIBLIOGRAPHIQUES}

ABADAN-UNAT, Nermin, et Neşe KEMİKSİz. 1986. Türk diş göçü 19601984. Yorumlu bibliografya (L'émigration internationale turque. Bibliographie commentée). Ankara, Ankara Üniversitesi. Bonn, Zentrum für Türkeistudien, $642 \mathrm{p}$.

AKHAN, Oya. 1992. "Femmes, familles et immigration turques : modification des pratiques de maternage ", dans Altay MANÇO et Ural MANÇO, éd. Turcs de Belgique. Identités et trajectoires d'une minorité. Bruxelles, Info-Türk : 115-156.

ALPAY, Şahin, et Hilmi SARIASLAN. 1984. Effects of Emigration. The Effects on the Town Kulu in Central Turkey of Emigration to Sweden. Stockholm, EIFO.

BASTENIER, Albert, et Felice DASSETTO. 1993. Immigration et espace public. La controverse de l'intégration. Paris, L'Harmattan, 285 p.

BOSKI, Pierre. 1994. *Vers une psychologie culturelle de l'identité nationale: approche théorique et empirique ", Cahiers internationaux de psychologie sociale, 24 : 54-75.

BOYER, Jean-Paul. 1983. "Processus d'installation d'une communauté turque à Terrasson, ville du Périgord ", Hommes et migrations, $1049: 36-47$.

CAMILLERI, Carmel, et al. 1990. Stratégies identitaires. Paris, Presses universitaires de France, 222 p.

DE TAPIA, Stéphane, éd. 1995. Turcs d'Europe et... d'ailleurs. Paris, INALCO, $534 \mathrm{p}$.

DEN EXTER, Jan, et Erol KUTLU. 1993. "Emirdağ : over de effecten van migratie op een Turks district ", Migrantenstudies, $4: 23-33$.

ERDER, Türköz, éd. 1984. Türkiye'de ailenin değişimi. Toplumbilimsel incelemeler (Le changement de la famille en Turquie. Recherches sociologiques). Ankara, Türk Sosyal Bilimler Derneği, 272 p.

ESTABLET, Roger. 1997. Comment peut-on être français ? 90 ouvriers turcs racontent. Paris, Fayard, 242 p.

GAILLY, Antoine. 1992. Content and Future Time Perspective of Motivational Goals of Belgian and Turkish Adolescents. Communication présentée au $13^{\mathrm{c}}$ congrès de l'International Association for Cross-Cultural Psychology, à Liège.

GITMEZ, S. Ali. 1983. Yurtdişina isçi göçü ve geri dönüşler. Beklentiler... Gerçekleşenler... (Immigration ouvrière à l'ètranger et 
les retours. Les attentes... les réalisations...). Istanbul, Alan Yayincilik, $215 \mathrm{p}$.

GÜVENÇ, Bozkurt. 1995. Türk kimliği (L'identité turque). Istanbul, Remzi Kitabevi, 448 p.

HALL, William. 1995. "Bati Avrupa'da Türk göçmenleri " (Immigrés Turcs en Europe occidentale), dans Margaret BAINBRIDGE, éd. Dünyada Türkler (Les Turcs dans le monde). Istanbul, Say Yayinlari : 325-338.

KADIOĞLU, Ayşe. 1994. "The impact of migration on gender roles : Findings of field research in Turkey ", International Migration, 22, $4: 533-560$.

KAĞITÇIBAŞI, Çiğdem. 1991. Insan, aile, kültür (L'humain, la famille et la culture). Istanbul, Remzi Kitabevi, 140 p.

KAĞITÇIBAŞI, Çiğdem, éd. 1982. Sex Roles, Family and Community in Turkey. Bloomington, Indiana University Press.

KÖKSAL, Sema. 1986. Refah toplumunda "getto" ve Türkler (Le "ghetto" et les Turcs dans la société d'opulence). Istanbul, Teknografik Matbaa, $190 \mathrm{p}$.

MANÇO, Altay, et Ural MANÇO. 1992. "Émigrations de Turquie : une grille de lecture des causes et des effets ", dans Altay MANÇO et Ural MANÇO, éd. Turcs de Belgique. Identités et trajectoires d'une minoritê. Bruxelles, Info-Türk : 9-26.

MANÇO, Altay. 1998. Valeurs et projets des jeunes issus de l'immigration. L'exemple des Turcs en Belgique. Paris, L'Harmattan, $157 \mathrm{p}$.

MANÇO, Altay. 1999. Développement socio-économique, structure des valeurs culturelles et poussée migratoire : résultats empiriques d'une étude exploratoire auprès de la population masculine de la région égéenne (Turquie). Document de travail, Liège, Institut de recherche, formation et d'action sur les migrations, $20 \mathrm{p}$.

MUCCHIELLI, Alex. 1985. Les Mentalités. Paris, Presses universitaires de France, 128 p.

NAESS, Roger. 1988. "Being an Alevi Muslim in South-western Anatolia and in Norway: Impact of migration on a heterodox Turkish community ", dans Georg LITHMAN et Elk GERHOLM, éd. The New Islamic Presence in Western Europe. Londres et New York, Mansell : 174-195.

OFFER, Daniel, et al. 1988. The Teenage World: Adolescents Selfimage in Ten Countries. New York, Plenum Press.

PHALET, Karen, et Willem CLAEYS. 1993. "A comparative study of Turkish and Belgian youths ", Journal of Cross-Cultural Psychology, $24,3: 319-343$.

PHALET, Karen, et Louk HAGENDOORN. 1996. "Personal adjustement to acculturative transitions: The Turkish experience ", International Journal of Psychology, 31, $2: 1-13$. 
RENIERS, Georges. 1997. On the Selectivity and Internal Dynamics of Labour Migration Processes: A Cross-cultural Analysis of Turkish and Morroccan Migration to Belgium. Communication présentée à la conférence internationale de l'European Association for Population Studies, à Cracovie.

SCHOORL, Jeanette. 1998. The Analysis of Factors Generating International Migration. A Multi-country Approach to Study the Determinants of Migration. Communication présentée au séminaire technique d'EUROSTAT sur le développement et les migrations internationales, tenu à La Haye.

SERTEL, Yildiz. 1987. Nord-sud: crise et immigration (le cas turc). Paris, Publisud, $285 \mathrm{p}$.

TIMMERMANN, Christine. 1992. "Turkish young women and the school system in Turkey and Belgium ", Migration, $15: 103-125$.

VAN DER ERF, Rob, et Lisbeth HEERING, éd. 1995. Causes of International Migration. Proceedings of a Workshop. Luxembourg, 14 16 December 1994. Bruxelles et Luxembourg, EUROSTAT, 274 p. 